Mon. Not. R. Astron. Soc. 000,111(2002) Printed 17 October $2018 \quad$ (MN LATEX style file v2.2)

\title{
Magnetic field geometry and chemical abundance distribution of the He-strong star CPD $-57^{\circ} 3509$
}

\author{
S. Hubrig ${ }^{1 \star}$, N. Przybilla ${ }^{2}$, H. Korhonen ${ }^{3}$, I. Ilyin ${ }^{1}$, M. Schöller ${ }^{4}$, S. P. Järvinen ${ }^{1}$, \\ M.-F. Nieva ${ }^{2}$, R.-D. Scholz ${ }^{1}$, S. Kimeswenger ${ }^{5,2}$, M. Ramolla ${ }^{6}$, A. F. Kholtygin ${ }^{7}$, \\ M. Briquet ${ }^{1,8}$ \\ ${ }^{1}$ Leibniz-Institut für Astrophysik Potsdam (AIP), An der Sternwarte 16, 14482 Potsdam, Germany \\ ${ }^{2}$ Institut für Astro- und Teilchenphysik, Universität Innsbruck, Technikerstr. 25/8, 6020 Innsbruck, Austria \\ ${ }^{3}$ Dark Cosmology Centre, Niels Bohr Institute, University of Copenhagen, Juliane Maries Vej 30, 2100 Copenhagen, Denmark \\ ${ }^{4}$ European Southern Observatory, Karl-Schwarzschild-Str. 2, 85748 Garching, Germany \\ ${ }^{5}$ Instituto de Astronomía, Universidad Católica del Norte, Avenida Angamos 0610, Casilla 1280, Antofagasta, Chile \\ ${ }^{6}$ Astronomisches Institut, Ruhr-Universität Bochum, Universitätsstr. 150, 44801 Bochum, Germany \\ ${ }^{7}$ Saint-Petersburg State University, Universitetskij pr. 28, Saint-Petersburg 198504, Russia \\ ${ }^{8}$ Institut d'Astrophysique et de Géophysique, Université de Liège, Allée du 6 Août, Bât. B5c, 4000 Liège, Belgium
}

Accepted ... Received ...; in original form ...

\begin{abstract}
The magnetic field of $\mathrm{CPD}-57^{\circ} 3509$ was recently detected in the framework of the BOB (B fields in OB stars) collaboration. We acquired low-resolution spectropolarimetric observations of $\mathrm{CPD}-57^{\circ} 3509$ with FORS 2 and high-resolution UVES observations randomly distributed over a few months to search for periodicity, to study the magnetic field geometry, and to determine the surface distribution of silicon and helium. We also obtained supplementary photometric observations at a timeline similar to the spectroscopic and spectropolarimetric observations. A period of $6.36 \mathrm{~d}$ was detected in the measurements of the mean longitudinal magnetic field. A sinusoidal fit to our measurements allowed us to constrain the magnetic field geometry and estimate the dipole strength in the range of 3.9-4.5 kG. Our application of the Doppler imaging technique revealed the presence of He I spots located around the magnetic poles, with a strong concentration at the positive pole and a weaker one around the negative pole. In contrast, high concentration Si III spots are located close to the magnetic equator. Further, our analysis of the spectral variability of CPD $-57^{\circ} 3509$ on short time scales indicates distinct changes in shape and position of line profiles possibly caused by the presence of $\beta$ Cep-like pulsations. A small periodic variability in line with the changes of the magnetic field strength is clearly seen in the photometric data.
\end{abstract}

Key words: Stars: abundances - Stars: evolution - Stars: magnetic field - Stars: massive - Stars: oscillations - Stars: individual: CPD $-57^{\circ} 3509$

\section{INTRODUCTION}

Recently, Przybilla et al. (2016) presented a firm detection of a mean longitudinal magnetic field of $\mathrm{kG}$ order in the early-B type star $\mathrm{CPD}-57^{\circ} 3509$, previously studied in a spectroscopic survey of massive stars in NGC 3293 by Evans et al. (2005). In their work, the authors focussed on the investigation of abundances, a model atmosphere, and the evolutionary state of the target in detail. The quantitative spectroscopic analysis of this star with the observed rather low $v \sin i$-value of $35 \mathrm{~km} \mathrm{~s}^{-1}$ yielded an effective tem-

* E-mail: shubrig@aip.de perature and a logarithmic surface gravity of $23750 \pm 250 \mathrm{~K}$ and $4.05 \pm 0.10$, respectively, and a surface helium fraction of $0.28 \pm 0.02$ by number (see also Maeder et al. 2014). The surface abundances of $\mathrm{C}, \mathrm{N}, \mathrm{O}, \mathrm{Ne}, \mathrm{S}$, and Ar were found to be compatible with the cosmic abundance standard (Nieva \& Przybilla 2012), whereas Mg, Al, Si, and Fe were depleted by about a factor of 2 . It was suggested that such an abundance pattern can be understood as the consequence of a fractionated stellar wind. Importantly, CPD $-57^{\circ} 3509$ with an elapsed main-sequence life time of about $50 \%$ has evolved significantly away from the zero-age main sequence and appears to be one of the most evolved He-strong stars known with an independent age constraint due to its clus- 
ter membership. Since the evolution of the magnetic field geometry across the main sequence in massive B-type stars is not well sampled in comparison to studies of magnetic fields of late-type $\mathrm{Bp}$ and intermediate-mass Ap stars, a detailed study of the magnetic field configuration and the surface chemical inhomogeneities in a significantly evolved magnetic He-strong star is of particular interest. Knowledge of the evolution of the magnetic field geometry, especially of the distribution of the obliquity angle $\beta$ (the orientation of the magnetic axis with respect to the rotation axis) is essential to understand the physical processes taking place in these stars and the origin of their magnetic fields. Further, although it is generally assumed that $\mathrm{CPD}-57^{\circ} 3509$ is a member of the open cluster NGC 3293, no careful study of its membership involving new data from Gaia was carried out yet. We review the membership status involving the available Gaia data in Appendix A

In order to characterise the properties of CPD $-57^{\circ} 3509$ in detail, we obtained time-series spectroscopy and spectropolarimetry with the FOcal Reducer low dispersion Spectrograph (FORS 2; Appenzeller et al. 1998) and the UVVisual Echelle Spectrograph (UVES; Dekker et al. 2000) to constrain the magnetic field geometry and reconstruct the distribution of silicon and helium on the stellar surface using Doppler Imaging (DI). Further, we carried out photometric observations using the $40 \mathrm{~cm}$ Bochum Monitoring Telescope (BMT; Ramolla et al. 2013) of the Cerro Armazones Observatory. In the first part of the paper we report on the period determination using magnetic and photometric data. In the second part we present the results of the application of the Doppler Imaging technique followed by the discussion of the surface element distribution with respect to the magnetic field configuration.

\section{OBSERVATIONS AND MAGNETIC FIELD MEASUREMENTS}

Fifteen FORS 2 spectropolarimetric observations of CPD $-57^{\circ} 3509$ were obtained in the framework of the ESO programme 094.D-0355 from 2014 November 17 to 2015 February 18, and further five within the framework of the ESO Large Programme 191.D-0255 during 2014 February and June, and 2015 March. The FORS 2 multi-mode instrument is equipped with polarisation analysing optics comprising super-achromatic half-wave and quarter-wave phase retarder plates, and a Wollaston prism with a beam divergence of $22^{\prime \prime}$ in standard resolution mode. We used the GRISM $600 \mathrm{~B}$ and the narrowest available slit width of $0^{\prime \prime} 4$ to obtain a spectral resolution of $R \sim 2000$. The observed spectral range from 3250 to $6215 \AA$ includes all Balmer lines, apart from $\mathrm{H} \alpha$, and numerous helium lines. For the observations, we used a non-standard readout mode with low gain $(200 \mathrm{kHz}, 1 \times 1$, low $)$, which provides a broader dynamic range, hence allowing us to reach a higher signal-to-noise ratio $(\mathrm{S} / \mathrm{N})$ in the individual spectra. The exposure time for each subexposure accounted for $5 \mathrm{~min}$. Each observation consisted of eight subexposures over approximately one hour including overheads.

Our first description of the assessment of longitudinal magnetic field measurements using FORS 1/2 spectropolarimetric observations was presented in several previous works (e.g. Hubrig et al. 2004a b, and references therein). To minimize the cross-talk effect and to cancel errors from different transmission properties of the two polarised beams, a sequence of subexposures at the retarder position angles $-45^{\circ}+45^{\circ},+45^{\circ}-45^{\circ},-45^{\circ}+45^{\circ}$, etc. is usually executed during the observations. Moreover, the reversal of the quarter wave plate compensates for fixed errors in the relative wavelength calibrations of the two polarised spectra. According to the FORS User Manual, the $V / I$ spectrum is calculated using:

$\frac{V}{I}=\frac{1}{2}\left\{\left(\frac{f^{\circ}-f^{\mathrm{e}}}{f^{\mathrm{o}}+f^{\mathrm{e}}}\right)_{-45^{\circ}}-\left(\frac{f^{\mathrm{o}}-f^{\mathrm{e}}}{f^{\circ}+f^{\mathrm{e}}}\right)_{+45^{\circ}}\right\}$

where $+45^{\circ}$ and $-45^{\circ}$ indicate the position angle of the retarder waveplate and $f^{\circ}$ and $f^{\mathrm{e}}$ are the ordinary and extraordinary beams, respectively. Rectification of the $V / I$ spectra was performed in the way described by Hubrig. Schöller \& Kholtvgin 2014a). Null profiles, N, are calculated as pairwise differences from all available $V$ profiles. From these, $3 \sigma$-outliers are identified and used to clip the $V$ profiles. This removes spurious signals, which mostly come from cosmic rays, and also reduces the noise. A full description of the updated data reduction and analysis will be presented in a separate paper (Schöller et al., in preparation; see also Hubrig. Schöller \& Kholtvgin 2014a). The mean longitudinal magnetic field, $\left\langle B_{\mathrm{z}}\right\rangle$, is measured on the rectified and clipped spectra based on the relation following the method suggested by Angel \& Landstreet (1970):

$\frac{V}{I}=-\frac{g_{\mathrm{eff}} e \lambda^{2}}{4 \pi m_{\mathrm{e}} c^{2}} \frac{1}{I} \frac{\mathrm{d} I}{\mathrm{~d} \lambda}\left\langle B_{\mathrm{z}}\right\rangle$,

where $V$ is the Stokes parameter that measures the circular polarization, $I$ is the intensity in the unpolarized spectrum, $g_{\text {eff }}$ is the effective Landé factor, $e$ is the electron charge, $\lambda$ is the wavelength, $m_{\mathrm{e}}$ is the electron mass, $c$ is the speed of light, $\mathrm{d} I / \mathrm{d} \lambda$ is the wavelength derivative of Stokes $I$, and $\left\langle B_{\mathrm{z}}\right\rangle$ is the mean longitudinal (line-of-sight) magnetic field.

The longitudinal magnetic field was measured in two ways: using the entire spectrum including all available lines, or using exclusively hydrogen lines. Furthermore, we have carried out Monte Carlo bootstrapping tests. These are most often applied with the purpose of deriving robust estimates of standard errors. The measurement uncertainties obtained before and after the Monte Carlo bootstrapping tests were found to be in close agreement, indicating the absence of reduction flaws. The results of our magnetic field measurements, those for the entire spectrum and only the hydrogen lines are presented in Table 1 where we also include in the first rows information about the previous magnetic field measurements presented by Przvbilla et al. (2016). The last row shows an additional measurement obtained on 2015 March 18 in the framework of the BOB ESO Large Programme 191.D-0255. The rotation phases presented in the last columns of Table 1 were calculated using the ephemeris determined from our period search described in Sect. 3 .

High resolution spectra of $\mathrm{CPD}-57^{\circ} 3509$ were obtained at the European Southern Observatory with the UVES high resolution spectrograph. The observations with an exposure time of about $40 \mathrm{~min}$ were obtained from 2014 December 6 to 2015 March 6 using the standard wavelength setting for dichroic mode (DIC-2437+760) with the $0.4^{\prime \prime}$ 
Table 1. Logbook of the FORS 2 polarimetric observations of CPD $-57^{\circ} 3509$, including the modified Julian date of mid-exposure followed by the achieved signal-to-noise ratio in the Stokes $I$ spectra around $5000 \AA$, and the measurements of the mean longitudinal magnetic field using the Monte Carlo bootstrapping test, only for the hydrogen lines and for all lines. In the last columns, we present the results of our measurements using the null spectra for the set of hydrogen and all lines and the phases calculated relative to a zero phase corresponding to a positive field extremum at MJD56983.5063 for the set with hydrogen lines assuming the rotation period $P_{\text {rot }}=6.36093 \mathrm{~d}$ and at MJD56983.5396 for the set with all lines assuming the rotation period $P_{\text {rot }}=6.36255 \mathrm{~d}$. All quoted errors are $1 \sigma$ uncertainties. Please note that the measurement marked by an asterisk on MJD 57027.3200 suffered from the fact that the guide star was lost several times due to thick clouds.

\begin{tabular}{|c|c|c|c|c|c|c|c|}
\hline MJD & $\mathrm{S} / \mathrm{N}$ & $\begin{array}{c}\left\langle B_{\mathrm{z}}\right\rangle_{\mathrm{hyd}} \\
{[\mathrm{G}]}\end{array}$ & $\begin{array}{c}\left\langle B_{\mathrm{z}}\right\rangle_{\text {all }} \\
{[\mathrm{G}]}\end{array}$ & $\begin{array}{c}\left\langle N_{\mathrm{z}}\right\rangle_{\text {hyd }} \\
{[\mathrm{G}]}\end{array}$ & $\begin{array}{c}\left\langle N_{\mathrm{z}}\right\rangle_{\text {all }} \\
{[\mathrm{G}]}\end{array}$ & $\varphi_{\text {hyd }}$ & $\varphi_{\text {all }}$ \\
\hline 56695.2463 & 1381 & $-287 \pm 126$ & $-23 \pm 60$ & $-377 \pm 139$ & $-101 \pm 64$ & 0.683 & 0.689 \\
\hline 56696.2876 & 1826 & $694 \pm 108$ & $539 \pm 51$ & $-116 \pm 104$ & $1 \pm 48$ & 0.846 & 0.853 \\
\hline 56810.0089 & 2025 & $-19 \pm 71$ & $88 \pm 54$ & $-28 \pm 86$ & $-45 \pm 59$ & 0.725 & 0.726 \\
\hline 56810.9983 & 2348 & $979 \pm 68$ & $920 \pm 48$ & $-108 \pm 77$ & $2 \pm 50$ & 0.880 & 0.882 \\
\hline 56978.3148 & 1397 & $966 \pm 159$ & $835 \pm 88$ & $-70 \pm 166$ & $31 \pm 94$ & 0.184 & 0.179 \\
\hline 57012.2145 & 943 & $-423 \pm 293$ & $-650 \pm 202$ & $-162 \pm 235$ & $23 \pm 220$ & 0.513 & 0.507 \\
\hline 57024.3376 & 1125 & $-588 \pm 237$ & $-815 \pm 137$ & $230 \pm 192$ & $211 \pm 121$ & 0.419 & 0.412 \\
\hline 57025.2844 & 1646 & $-590 \pm 127$ & $-557 \pm 75$ & $-87 \pm 132$ & $5 \pm 74$ & 0.568 & 0.561 \\
\hline 57026.2200 & 1127 & $24 \pm 174$ & $32 \pm 105$ & $178 \pm 163$ & $107 \pm 100$ & 0.715 & 0.708 \\
\hline $57027.3200^{*}$ & 665 & $-106 \pm 384$ & $-62 \pm 199$ & $237 \pm 331$ & $145 \pm 202$ & 0.888 & 0.881 \\
\hline 57030.2079 & 1406 & $-627 \pm 138$ & $-444 \pm 94$ & $249 \pm 157$ & $89 \pm 105$ & 0.342 & 0.335 \\
\hline 57031.3116 & 1507 & $-1052 \pm 121$ & $-847 \pm 73$ & $-65 \pm 141$ & $-82 \pm 81$ & 0.515 & 0.508 \\
\hline 57039.3509 & 1472 & $347 \pm 140$ & $272 \pm 77$ & $-6 \pm 134$ & $35 \pm 71$ & 0.779 & 0.772 \\
\hline 57057.2937 & 1835 & $-666 \pm 107$ & $-508 \pm 64$ & $-87 \pm 114$ & $-18 \pm 63$ & 0.600 & 0.592 \\
\hline 57059.1600 & 1217 & $709 \pm 173$ & $791 \pm 98$ & $-169 \pm 188$ & $-14 \pm 104$ & 0.893 & 0.885 \\
\hline 57060.1721 & 1318 & $1209 \pm 157$ & $1122 \pm 104$ & $-8 \pm 170$ & $-20 \pm 99$ & 0.053 & 0.044 \\
\hline 57069.0658 & 1343 & $-1021 \pm 164$ & $-787 \pm 110$ & $4 \pm 175$ & $119 \pm 118$ & 0.451 & 0.442 \\
\hline 57070.0817 & 1175 & $-818 \pm 158$ & $-606 \pm 136$ & $257 \pm 195$ & $-61 \pm 132$ & 0.610 & 0.602 \\
\hline 57071.0569 & 1086 & $127 \pm 202$ & $212 \pm 118$ & $30 \pm 182$ & $-74 \pm 105$ & 0.764 & 0.755 \\
\hline 57099.0931 & 1826 & $582 \pm 99$ & $671 \pm 62$ & $-75 \pm 101$ & $-33 \pm 61$ & 0.171 & 0.162 \\
\hline
\end{tabular}

Table 2. Logbook of the UVES observations. The phases were calculated relative to a zero phase corresponding to a positive field extremum at MJD56983.5396 for the set with all lines assuming the rotation period $P_{\text {rot }}=6.36255 \mathrm{~d}$

\begin{tabular}{cccc}
\hline \hline MJD & Date & Phase & S/N \\
\hline 56997.27269 & $2014 / 12 / 06$ & 0.159 & 175 \\
57000.30922 & $2014 / 12 / 09$ & 0.636 & 223 \\
57023.20634 & $2015 / 01 / 01$ & 0.234 & 189 \\
57025.19286 & $2015 / 01 / 03$ & 0.547 & 168 \\
57047.23708 & $2015 / 01 / 25$ & 0.011 & 249 \\
57052.20926 & $2015 / 01 / 30$ & 0.793 & 191 \\
57055.07998 & $2015 / 02 / 02$ & 0.244 & 228 \\
57056.15139 & $2015 / 02 / 03$ & 0.412 & 255 \\
57082.06466 & $2015 / 03 / 01$ & 0.485 & 244 \\
57087.14368 & $2015 / 03 / 06$ & 0.284 & 227 \\
\hline
\end{tabular}

slit in the blue arm giving a spectral resolving power of $R \sim 80,000$ and the $0.3^{\prime \prime}$ slit in the red arm to achieve a resolution of $R \sim 110,000$. The wavelength coverage was $3730-9390 \AA$ with a gap between 5000 and $5700 \AA$. Initially, we asked for 20 observations in service mode, but, unfortunately, only ten observations were executed. The summary of the UVES spectroscopic observations is presented in Table 2. The table gives the Modified Julian date at the middle of each observation, the observing date, the rotational phase, and the $\mathrm{S} / \mathrm{N}$. The $\mathrm{S} / \mathrm{N}$ is given per resolution element and is measured from the spectral region around $4630 \AA$. All observations were phased using the ephemeris
HJD $=2456983.5396+6.36255 \times E$, referring to the time of the maximum positive magnetic field. Advantageously, the phase distribution of the obtained spectra appears suitable for a Doppler imaging analysis to study the surface distribution of silicon and helium, which exhibit the most distinct spectrum variability in He-strong stars.

Supplementary photometric observations were carried out at the BMT. Taken in 2014 April and May and during 2015 March 31 to April 4, the photometry covers a timeline similar to the spectroscopic and spectropolarimetric observations. Johnson $B$ and $V$ filters were used and a $3 \times 3$ dithering pattern of observations were obtained each night to improve the sampling and photometric accuracy. Seven photometrically invariable cluster stars (numbers 3, 44, 45, 51, 58, 97, and 110 of Baume et al. 2003) in the vicinity of $\mathrm{CPD}-57^{\circ} 3509$ were used for comparison in differential photometry. They covered a magnitude range of $9.4 \leqslant$ $\left(\mathrm{m}_{\mathrm{V}}, \mathrm{m}_{\mathrm{B}}\right) \leqslant 12^{\mathrm{m}} 9$ and colors from $0.06 \leqslant(\mathrm{~B}-\mathrm{V}) \leqslant 0.27$, thus well enclosing the target and minimizing filter effects. The rms between the comparison stars were $\sigma_{\mathrm{V}}=0$. 0047 and $\sigma_{\mathrm{B}}=0 . \mathrm{m}^{\mathrm{m}} 0062$. The photometric data are summarized in Tables B1 and B2 presented in Appendix B. Our photometric zero values (median of the 2014 data points) for $\mathrm{CPD}-57^{\circ} 3509$ are $B=10.80 \pm 0.01$ and $V=10.69 \pm 0.01$.

\section{PERIOD DETERMINATION}

The results of our frequency analysis based on the longitudinal magnetic field measurements presented in Table 1 and 

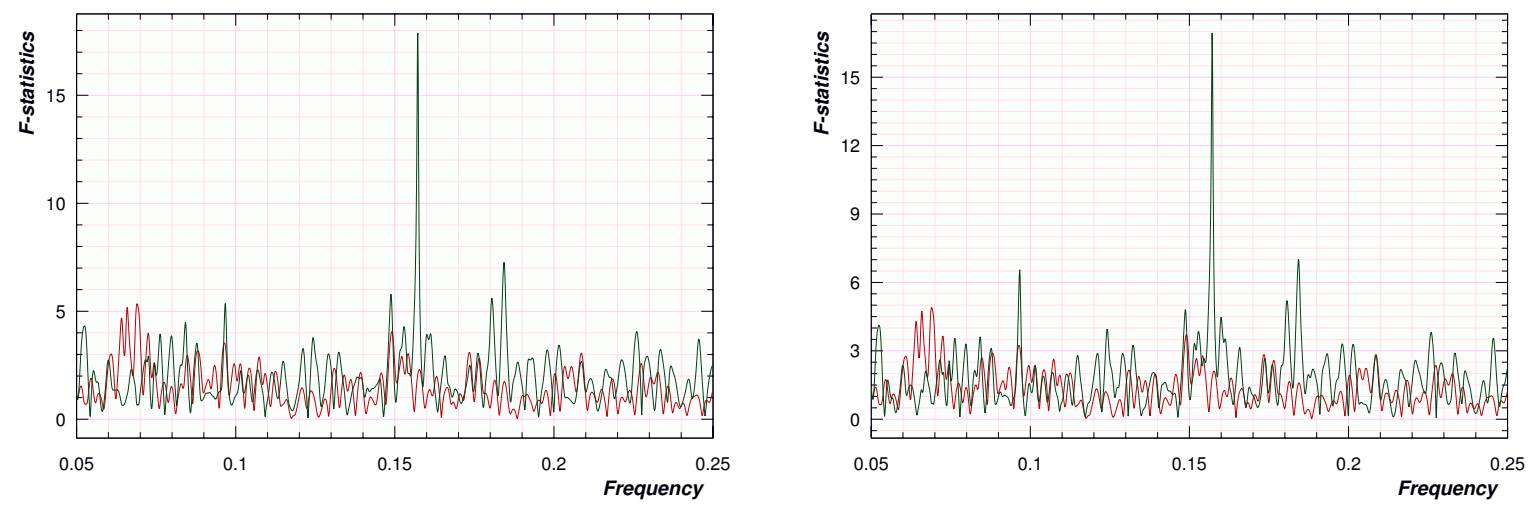

Figure 1. Left panel: F-statistics frequency spectrum $\left(\right.$ in $\left.^{-1}\right)$ for the longitudinal magnetic field measurements of $\mathrm{CPD}-57^{\circ} 3509$ using the hydrogen lines. Right panel: F-statistics frequency spectrum using the entire spectrum for the measurements. The window function is indicated by the red color.

performed using a non-linear least squares fit to the multiple harmonics utilizing the Levenberg-Marquardt method (Press et al. 1992) are presented in Fig. 11 To detect the most probable period, we calculated the frequency spectrum and for each trial frequency we performed a statistical F-test of the null hypothesis for the absence of periodicity (Seber 1977). The resulting F-statistics can be thought of as the total sum including covariances of the ratio of harmonic amplitudes to their standard deviations, i.e. a signal-to-noise ratio. The highest peak in the frequency spectrum for the hydrogen lines not coinciding with the window function corresponds to a period of $6.36093 \pm 0.00026 \mathrm{~d}$ and that for the measurements of the entire spectrum corresponds to a period of $6.36255 \pm 0.00026 \mathrm{~d}$. We note that taking into account the precision of the period determination, the difference between the two periods is not significant. On the other hand, as the achieved magnetic field measurement accuracy is higher for the set using all lines, the rotation period of $6.36255 \mathrm{~d}$ identified from these measurements is expected to be more reliable and is preferred in the following discussion on the surface element distribution in Sect. 4

In Fig. 2 we present all measurements, those using the entire spectrum and those using only the hydrogen lines, phased with the corresponding rotation periods and the best sinusoidal fits calculated for these measurements. As already mentioned in the caption of Table 1 the deviating point close to the phases 0.881 , respective 0.888 , is caused by the unfavourable weather during the observations at that phase.

Since CPD $-57^{\circ} 3509$ already finished half of its mainsequence lifetime (Przybilla et al. 2016) and is already passing through the $\beta$ Cep instability strip, we checked the stability of the Stokes $I$ spectral lines over the full sequences of sub-exposures obtained on a time scale of tens of minutes. Along with different radial velocity shifts of lines belonging to different elements, we also detect distinct changes in line profiles taking place on time-scales corresponding to the duration of the sub-exposure sequences in the individual observations. In Fig. 3. we present the behaviour of the line profiles in individual spectral lines. The time difference between subexposures accounts for about $20 \mathrm{~min}$. However, with the current data we cannot identify the periodicity of the detected variability, which is probably caused by the presence of $\beta$ Cep-like pulsations. Thus, future observations should focus on the careful search for periodicity and on the identification of the pulsation modes.

The periodicity derived from spectropolarimetry (and the corresponding spottiness, see below) plus the tentative $\beta$ Cep-like pulsations are expected to be detectable in the photometric data. Our differential photometry data in $B$ and $V$ are displayed in Fig. 4, phased according to the period obtained from the magnetic analysis using only the hydrogen lines - the behaviour is very similar for the case of the phasing based on all lines. A small periodic variability in line with the changes of the magnetic field strength is clearly seen. The dispersion of the higher-cadence data of 2015 (taken within $\sim 2 / 3$ of the rotation period) could be interpreted in favour of the presence of $\beta$ Cep pulsations, but this is close to the detection limit and requires dedicated follow-up observations for confirmation. Finally, we want to note a difference in the mean $B$ and $V$ magnitudes from the present work and that of Baume et al. (2003), which are $0 .{ }^{\mathrm{m}} 07$ and 0.06 fainter than our values. As the comparison stars used for the differential photometry show only an rms of 0 . 02 between both studies (data taken $\sim 20$ years apart), this may imply some long-term variability for $\mathrm{CPD}-57^{\circ} 3509$ as well.

\section{DOPPLER IMAGING USING UVES OBSERVATIONS}

As we mentioned above, the rotational phase coverage and $\mathrm{S} / \mathrm{N}$ of the UVES observations is quite good and enables mapping the chemical element patterns, in particular of the most strongly varying elements $\mathrm{He}$ and $\mathrm{Si}$. The phase coverage of the observations is not optimal, though, and four observations have been obtained close in phase. Still, the largest phase gap is 0.22 , and occurs between phases 0.79 and 0.01 . Other areas on the stellar surface are well covered, and the phase gaps are less than 0.15 , posing no problems for the Doppler imaging technique. Tests show that phase gaps as large as about 0.28 do not significantly affect the reconstruction of the features (Rice \& Strassmeier 2000) if the $\mathrm{S} / \mathrm{N}$ is good. Commonly seen problems caused by non-perfect phase coverage are the blurring of features and not being able to recover small spots (see e.g., 

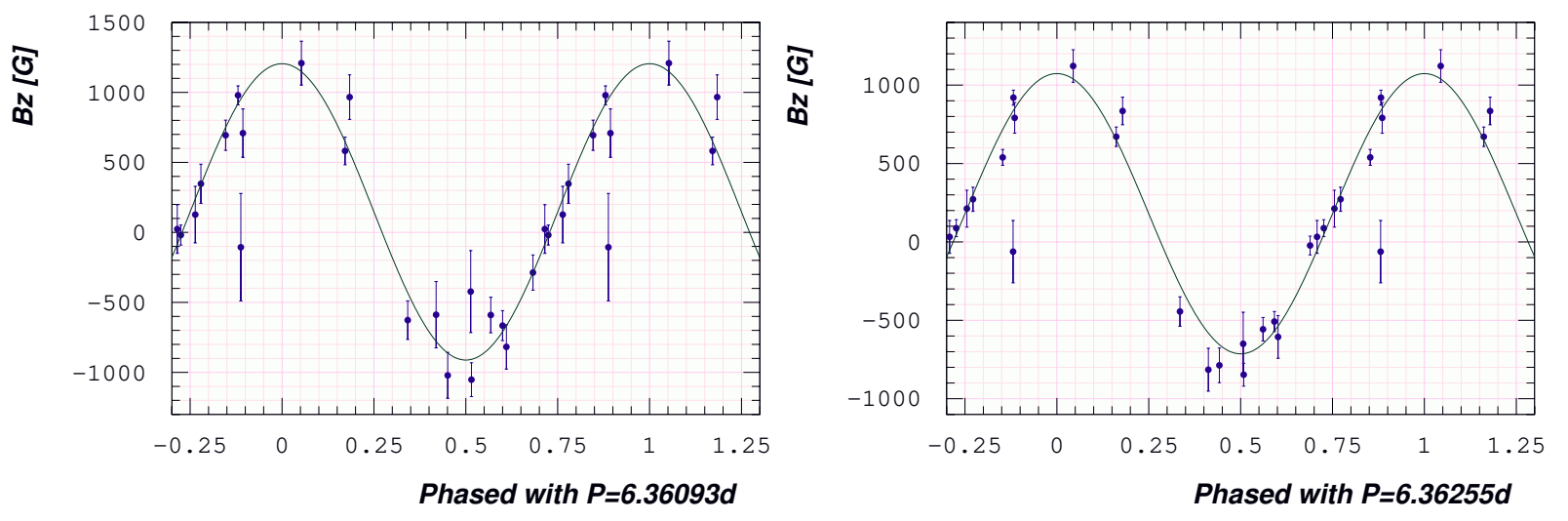

Figure 2. Left panel: Longitudinal magnetic field variation of $\mathrm{CPD}-57^{\circ} 3509$ measured using the hydrogen lines phased with the $6.36093 \mathrm{~d}$ period. The solid line represents a fit to the data with a mean value for the magnetic field of $\overline{\left\langle B_{\mathrm{z}}\right\rangle}=147 \pm 52 \mathrm{G}$, and an amplitude of $A_{\left\langle B_{\mathrm{z}}\right\rangle}=1058 \pm 64 \mathrm{G}$. For the presented fit, we assume a zero phase corresponding to a positive field extremum at MJD56983.5063 \pm 0.0590 . Right panel: Longitudinal magnetic field variation of CPD $-57^{\circ} 3509$ measured using the entire spectrum phased with the $6.36255 \mathrm{~d}$ period. The solid line represents a fit to the data with a mean value for the magnetic field of $\overline{\left\langle B_{\mathrm{z}}\right\rangle}=$ $180 \pm 47 \mathrm{G}$ and an amplitude of $A_{\left\langle B_{\mathrm{z}}\right\rangle}=894 \pm 57 \mathrm{G}$. For this fit, we assume a zero phase corresponding to a positive field extremum at $M J D 56983.5396 \pm 0.0646$. Please note that values below 0 and above 1 are repetitions and plotted to visualize the transition at the intervall borders.
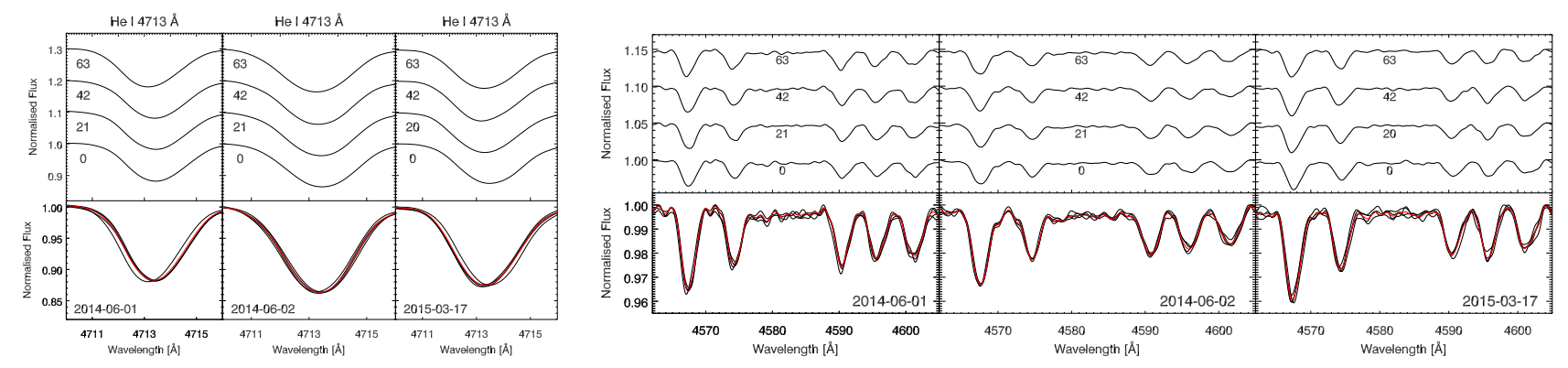

Figure 3. Left panel: The behaviour of He I 4713 in the FORS 2 spectra in each individual subexposure belonging to observations on three different epochs. For each epoch, in the upper row, we present the line profiles shifted in vertical direction for best visibility. The time difference (in minutes) between the subexposure and the beginning of the observations is given close to each profile. The lower row shows all profiles overplotted. The average profile is indicated by the red line. Right panel: The same as in the left panel, but for the metallic lines in the spectral region $4562-4605 \AA$.

Collier-Cameron \& Unruh 1994). Chemical spots are large structures and therefore can easily be mapped with the phase coverage of the current observations. Naturally, one should be careful when interpreting the results, especially small features, in the location of the largest phase gap. In Sect. 4.2 the discussion includes the possible impact of the phase gap on the obtained maps.

Since observations with HARPSpol are usually carried out in visitor mode, we do not have high-resolution spectropolarimetric observations of $\mathrm{CPD}-57^{\circ} 3509$ on our disposal. The magnetic field strength is however only a few $\mathrm{kG}$, and as we show below, it is possible to use the Doppler imaging technique to obtain an approximate elemental distribution without taking into account the impact of the magnetic field on the line profiles. The $\mathrm{He}$ mapping is carried out using HeI 4713. For Si III, three lines located close to each other, 4552.622, 4567.840, and $4574.757 \AA$, were used simultaneously. For the phase calculation we employed the rotation period of $6.36255 \pm 0.00026 \mathrm{~d}$ identified in our magnetic field measurements using the entire spectrum. The surface abundance maps were obtained with the INVERS7PD inversion code, which was originally developed by Piskunov, Tuominen \& Vilhu (1990) and modified by Hackman. Jetsu \& Tuominen (2001). INVERS7PD compares the observations to a grid of synthetic local line-profiles, which were calculated using the SPECTRUM spectral synthesis code (Grav \& Corbally 1994) and ATLAS9 stellar atmospheres by Kurucz (1993). The He I line properties are hard coded into SPECTRUM, and the Si III line parameters were originally obtained from VALD (Piskunov et al. 1995; Kupka et al. 1999). When using the Si abundance determined by Przvbilla et al. (2016), the three Si III lines were on average well fitted, but the $4552.622 \AA$ Aine was slightly too weak, while the $4574.7570 \AA$ line appeared slightly too strong. To minimise the influence of the errors in the atomic data, we followed the standard procedure and changed the oscillator strengths of the lines to improve the overall fit (see e.g., Makaganiuk et al. 2011; Korhonen et al. 2013). The changes needed were small and allowed for fitting all three Si III lines simultaneously. Table 3 gives the parameters used for these lines.

For the model calculations, 20 limb angles were used together with the stellar parameters determined by Przvbilla et al. 2016): $T_{\text {eff }}=23750 \mathrm{~K}, \log g=4.0$, and 


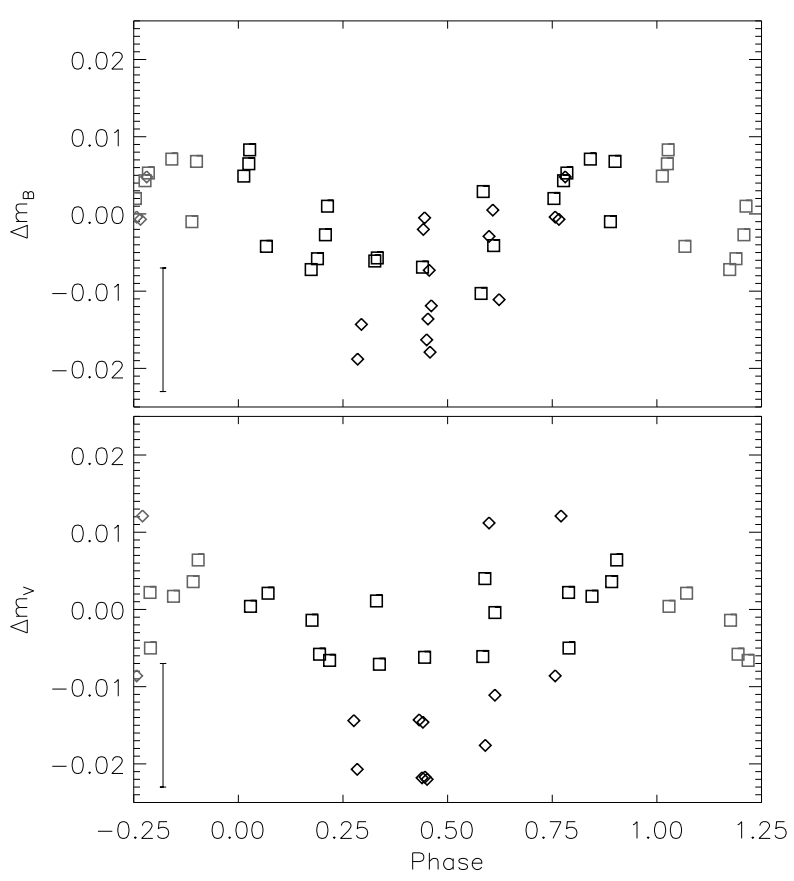

Figure 4. Differential photometry of CPD $-57^{\circ} 3509$ in the $B$ (upper panel) and $V$ (lower panel) bands phased with the period from the magnetic field analysis using the hydrogen lines. Data from 2014 are displayed as boxes, data from 2015 as diamonds. A (conservative) error bar for the individual measurements is shown at the bottom left in each panel. Please note that values below 0 and above 1 are repetitions and plotted to visualize the transition at the intervall borders.

Table 3. Si III $\log g f$ values used in this work.

\begin{tabular}{crr}
\hline \hline $\begin{array}{c}\text { Wavelength } \\
{[\AA]}\end{array}$ & $\begin{array}{c}\log g f \\
\text { VALD }\end{array}$ & \multicolumn{1}{c}{$\begin{array}{c}\log g f \\
\text { this work }\end{array}$} \\
\hline 4552.6220 & 0.181 & 0.275 \\
4567.8400 & -0.039 & -0.039 \\
4574.7570 & -0.509 & -0.709 \\
\hline
\end{tabular}

micro- and macroturbulence of 2.0 and $10.0 \mathrm{~km} \mathrm{~s}^{-1}$, respectively. From the inversions, we obtained a best fit inclination angle of $58 \pm 10^{\circ}$. Notably, the Doppler imaging technique is very sensitive to the $v \sin i$ and the inclination angle values in the sense that no converging solution can be found if these values are under- or overestimated.

Przybilla et al. (2016) estimated the $v \sin i$ value of $\mathrm{CPD}-57^{\circ} 3509$ to be $35 \pm 2 \mathrm{~km} \mathrm{~s}^{-1}$. In the inversions, the best fit from the SiIII lines was obtained for $v \sin i=$ $34.5 \mathrm{~km} \mathrm{~s}^{-1}$, in excellent agreement with the results of Przybilla et al. (2016). On the other hand, for the He I line, the best fit was obtained for $v \sin i=26.5 \mathrm{~km} \mathrm{~s}^{-1}$, which is lower than the other estimates. There are several possible reasons for this discrepancy. First, weaker metal lines are more sensitive to $v \sin i$ values and broad He I lines dominated by Stark effect are usually avoided in the determination of rotation rates. Si III and He I lines also have dif- ferent Landé factors. Further, the star is likely a $\beta$ Ceplike pulsator. Depending on the type of pulsations, this can have more impact on the Si III lines rather than on the He I lines, causing stronger line broadening in the Si III lines. We also note that similar discrepancies in the determination of $v \sin i$ values for different elements were mentioned in other works, which were using imaging methods (e.g. Yakunin et al. 2015). We also remark that SPECTRUM is verified to work at the spectral type range $\mathrm{B}$ to mid-M, making CPD $-57^{\circ} 3509$ close to the limit of the code's capabilities. Furthermore, SPECTRUM does not use NLTE in the spectral synthesis, and HeI is more sensitive to departures from NLTE than Si III. For these reasons, the absolute abundance scales in the obtained maps are not necessarily precise. Still, this will not affect the relative abundances of the spots, nor their locations.

\subsection{Magnetic field geometry}

The simplest model for a magnetic field geometry is based on the assumption that the studied stars are oblique dipole rotators, i.e., their magnetic field can be approximated by a dipole with its magnetic axis inclined with respect to the rotation axis.

From the variation of the phase curve for the field measurements with a mean of $\overline{\left\langle B_{\mathrm{z}}\right\rangle}=180 \pm 47 \mathrm{G}$ and an amplitude of $A_{\left\langle B_{\mathrm{z}}\right\rangle}=894 \pm 57 \mathrm{G}$, we calculate $\left\langle B_{\mathrm{z}}\right\rangle^{\mathrm{min}}=$ $-714 \pm 74 \mathrm{G}$ and $\left\langle B_{\mathrm{z}}\right\rangle^{\max }=1074 \pm 74 \mathrm{G}$. Using the definition by Preston (1967)

$r=\frac{\left\langle B_{\mathrm{z}}\right\rangle^{\min }}{\left\langle B_{\mathrm{z}}\right\rangle^{\max }}=\frac{\cos \beta \cos i-\sin \beta \sin i}{\cos \beta \cos i+\sin \beta \sin i}$,

we find $r=-0.665 \pm 0.075$ and finally following

$\beta=\arctan \left[\left(\frac{1-r}{1+r}\right) \cot i\right]$,

and employing $i=58 \pm 10^{\circ}$ obtained from our Doppler imaging inversions, we calculate a magnetic obliquity angle $\beta=72 \pm 8^{\circ}$. Assuming a limb-darkening coefficient $u=0.3$, typical for stars with $T_{\text {eff }}=23750 \mathrm{~K}$ (Przybilla et al. 2016), we estimate a dipole strength of $3.91 \pm 0.36 \mathrm{kG}$ using the model by Stibbs (1950), as formulated by Preston (1967):

$B_{\mathrm{d}}=\left\langle B_{\mathrm{z}}\right\rangle^{\max }\left(\frac{15+u}{20(3-u)}(\cos \beta \cos i+\sin \beta \sin i)\right)^{-1}$.

Using the parameters of the sinusoidal fit to the values resulting from only the hydrogen lines $\left(\overline{\left\langle B_{\mathrm{z}}\right\rangle}=147 \pm 52 \mathrm{G}\right.$ and $A_{\left\langle B_{\mathrm{z}}\right\rangle}=1058 \pm 64 \mathrm{G}$ ), we obtain slightly different values for the magnetic field model: $r=-0.757 \pm 0.077, \beta=78 \pm 6^{\circ}$, and $B_{\mathrm{d}}=4.51 \pm 0.45 \mathrm{kG}$. Given the size of the errors of the dipole strength determination, the difference in the derived values of the dipole strengths is not significant.

\subsection{Abundance maps and comparison to the magnetic pole location}

The He I and Si III distributions are shown in Fig. 5, and the model fits to the observations are given in Fig. 6 The locations of the chemical spots are very similar in both maps, but the behaviour is opposite. The main concentration of He I occurs at phase 0.0 , and a weaker concentration around 

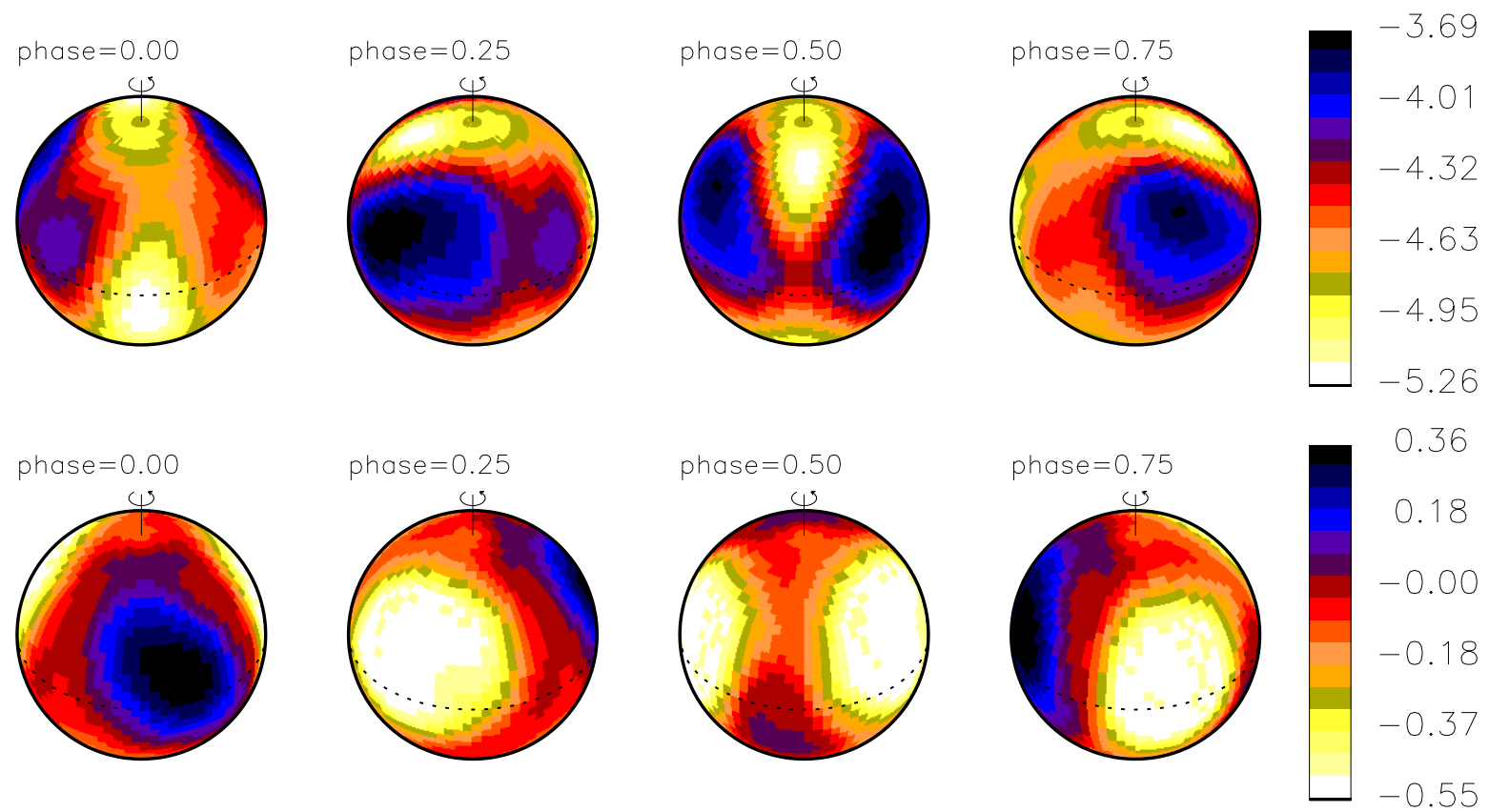

Figure 5. Chemical abundance maps of $\mathrm{CPD}-57^{\circ} 3509$ for SiIII (top) and He I 4713 (bottom). The Si III map has been obtained simultaneously from three lines: Si III 4553, Si III 4568, and Si III 4575. The surface distribution of both elements is shown for four different phases that are 0.25 apart. The abundance is given with respect to the total number density of atoms and ions.

the phase 0.5. The He I underabundance spots are located at phases 0.4 and 0.7 . In contrast, the main concentrations of Si III occur around the phases 0.4 and 0.7 , and the underabundance spots are located around the phases 0.0 and 0.5. In Si III, also a third smaller overabundance spot is seen around the phase 0.1 . Some indication of a corresponding spot could also be seen around phase 0.9 , but due to the phase gap in the observations at phases $0.78-0.01$, we cannot verify the reality of this potential fourth Si III spot.

Figure 7 shows the locations of the magnetic poles together with the abundance maps. The HeI overabundance spots clearly occur around the magnetic poles, with a stronger concentration at the positive pole and a weaker one around the negative pole. The locations do not coincide completely in phase with the magnetic poles, and the overabundance spots seem to be shifted some 0.1 in phase away from the poles. On the other hand, Si III shows underabundance around the magnetic poles, and the main overabundance spots fall closer to the magnetic equator. Interestingly, it seems that the Si III concentrations occur somewhat shifted towards the negative pole, not at the magnetic equator itself. Similarly, the underabundance spots of He I are located closer to the negative magnetic pole.

The presented Doppler maps for $\mathrm{Si}$ and He support the dipole-dominated magnetic topology of CPD $-57^{\circ} 3509$. On the other hand, the presence of a third $\mathrm{Si}$ spot detected around the phase 0.1 and considerable abundance and field strength differences between the two magnetic poles suggest the contribution of a non-negligible higher-order magnetic multipole. Previous studies of upper main-sequence stars showed that inhomogeneous chemical abundance distributions are only observed on the surface of magnetic chemically peculiar Ap and Bp stars with large-scale organised magnetic fields. In these stars, the abundance distribu- tion of certain elements is non-uniform and non-symmetric with respect to the rotation axis. The majority of studies of $\mathrm{Ap}$ and $\mathrm{Bp}$ stars have revealed a kind of symmetry between the topology of the magnetic field and the element distribution (see e.g. Rice. Whelau \& Holmgren 1997; Yakunin et al. 2015; Hubrig et al. 2014b). Thus, the structure of the magnetic field can be studied by producing the surface element maps and measuring the magnetic field using spectral lines of inhomogeneously distributed elements separately. However, due to the low resolution of our FORS 2 spectra, we are not able to study the detailed surface magnetic field distribution. Furthermore, only the availability of high-resolution spectra in all four Stokes parameters would allow us to obtain self-consistent mapping of spots and magnetic fields by means of Zeeman Doppler imaging (ZDI; e.g. Brown et al. 1991).

\section{DISCUSSION}

Our spectropolarimetric monitoring of CPD $-57^{\circ} 3509$ using FORS 2 at the VLT shows the presence of an approximately dipolar magnetic field with a polar strength of $4 \mathrm{kG}$, reversing over the rotation period of $6.36 \mathrm{~d}$. Using the Doppler imaging technique, we were able to constrain the inclination of the rotation axis to the line of sight, $i=58 \pm 10^{\circ}$, and estimate the obliquity of the magnetic axis, $\beta=72 \pm 8^{\circ}$. In the past it was considered neither theoretically nor observationally how the magnetic field geometry in massive B-type stars evolves across the main sequence. Thus, the analysis of the magnetic field configuration of CPD $-57^{\circ} 3509$ is of special interest as it is one of the most evolved He-strong stars currently known. Hubrig, North \& Schöller (2007) studied the evolution of the magnetic field geometry in late B-type 

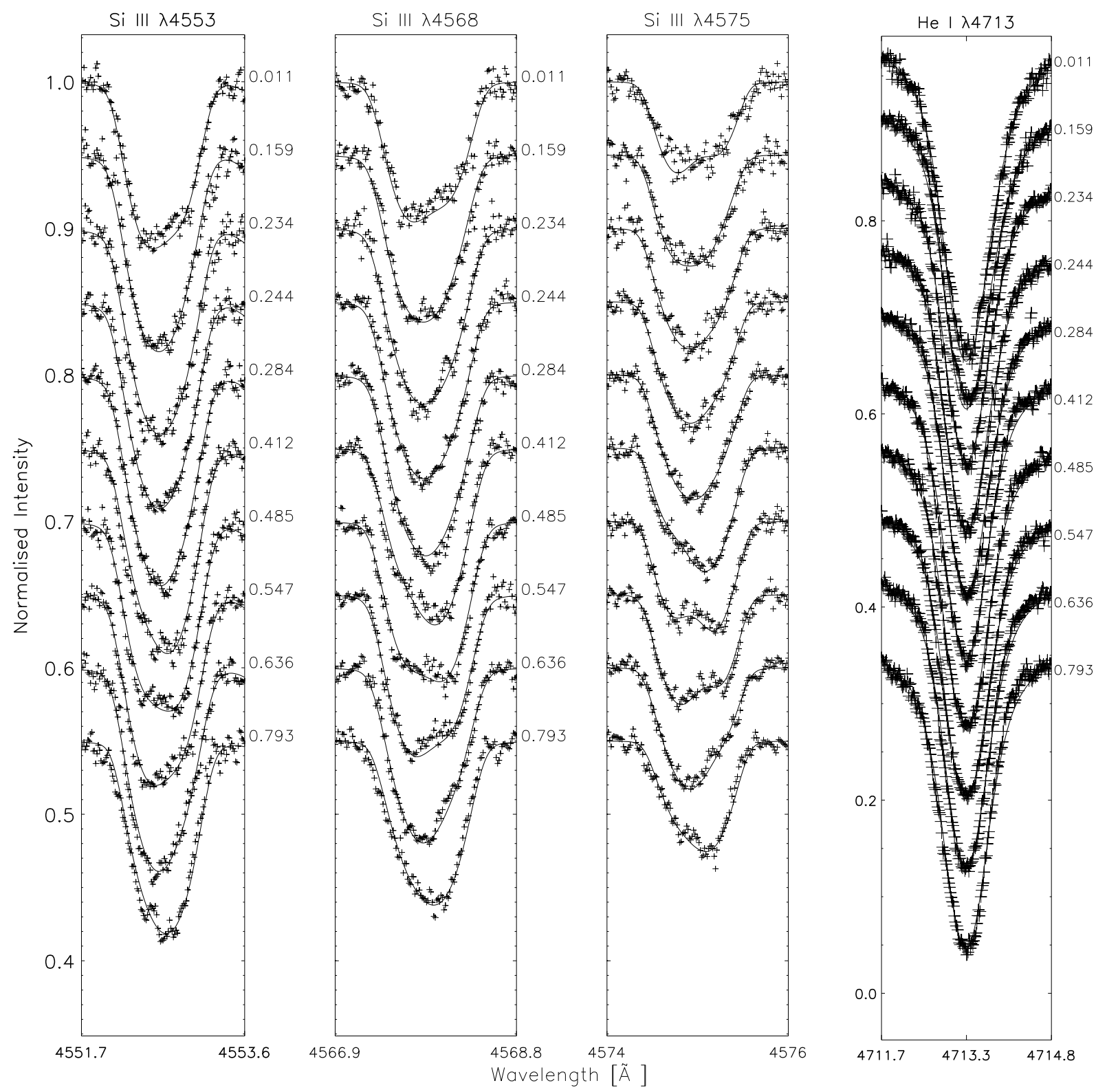

Figure 6. Spectroscopic observations (plus signs) together with the model fit from the inversions (solid line). The fits are shown for all the lines used in the inversions (from left to right): Si III 4553, Si III 4568, Si III 4575, and He I 4713. Line profiles are shifted in vertical direction for better visibility.

stars with masses $\leqslant 5 M_{\odot}$ with accurate Hipparcos parallaxes and definitely determined longitudinal magnetic fields. They found that the distribution of relative ages peaks at the ZAMS, with two secondary lower peaks at the relative ages around $60 \%$ and $80 \%$. The strongest magnetic fields were found in younger stars in terms of the elapsed fraction of their main-sequence life. Further, rotation periods of lateB type stars slightly increase with age, which is consistent with the assumption of conservation of angular momentum during their life on the main sequence, without any hint of a braking mechanism (see also North \& Cramer 1984; North 1985). The fact that the strongest magnetic fields are only observed close to the ZAMS can be interpreted as a magnetic field decay in stars at advanced ages. As for the magnetic field geometry, the authors detected a strong hint for an in- crease of obliquity $\beta$ with elapsed time on the main sequence. Moreover, $21 \%$ out of the studied 33 stars have magnetic phase curves fitted by a double wave, indicating that the magnetic topology in late-B type stars is frequently more complex than just a single dipole. Obviously, a comparison of the evolution of magnetic field geometries between the higher-mass He-strong stars and the lower-mass magnetic B-type stars is urgently needed to constrain the mechanism of the magnetic field generation in such stars.

There is also a large dissimilarity between the lower mass magnetic Ap stars and the He-strong stars in respect to the orientation of their magnetic axes and the period lengths. The results of modeling a small sample of Ap stars by Landstreet \& Mathys (2000) implied that stars with small obliquity values $\beta$ of the model magnetic axis to the 


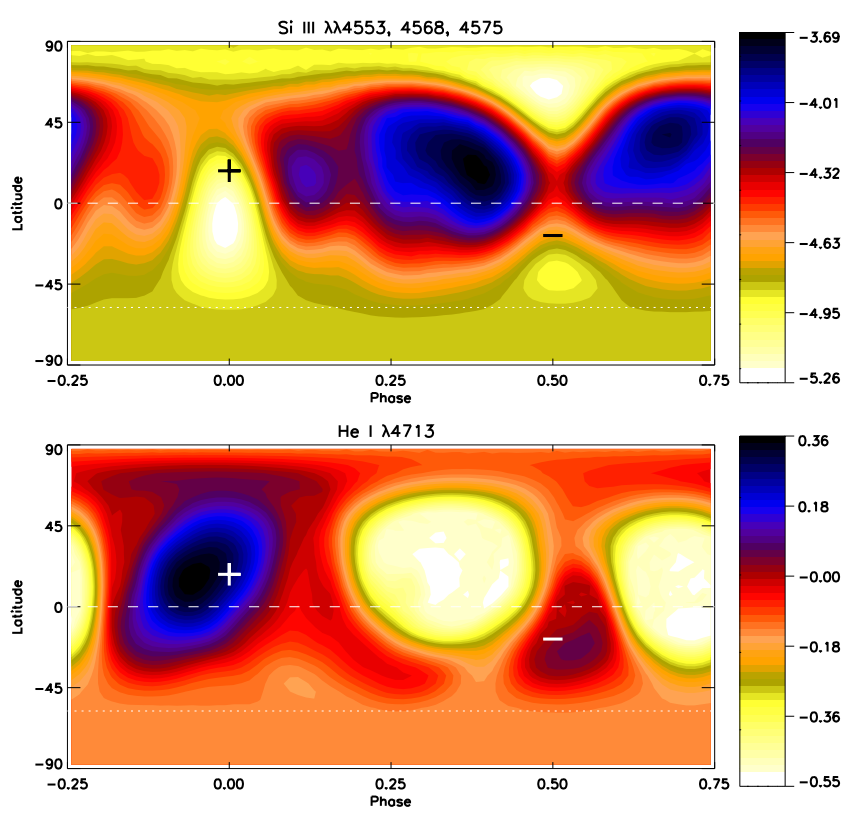

Figure 7. Mercator abundance maps plotted together with the location of the magnetic poles. As before, Si III is plotted at the top and He I at the bottom. The plus-sign denotes the location of the positive magnetic pole, and the negative pole is given by the minus-sign. The dashed line shows the stellar rotational equator, and the dotted line gives the limit below which the star is not seen due to its inclination. Note that the phases go from -0.25 to 0.75 to better show the area around the magnetic poles.

rotation axis, of the order of $20^{\circ}$, have periods longer than 25 days. However, recent studies of the magnetic field geometries in He-strong stars do not confirm this trend: a number of fast rotating He-strong stars with periods below $2 \mathrm{~d}$ show low obliquities of their magnetic axes (e.g. Grunhut et al. 2012; Sikora et al. 2015; Hubrig et al. 2017).

The study of the variability of the $\mathrm{Si}$ and He lines showed the presence of significant chemical abundance variations across the stellar photosphere. The location of the chemical spots is roughly correlated with the topology of the magnetic field, where the main concentration of He is observed in the vicinity of the positive magnetic pole and Si underabundant spots around the poles. Only three mapping studies were devoted to He-strong stars in the past, all of them using ZDI (Yakunin et al. 2015; Oksala et al. 2015; Kochukhov et al. 2011). Yakunin et al. (2015) studied the $\mathrm{He}$ and $\mathrm{O}$ abundance distribution on the surface of the He-strong star HD 184927. Similar to our DI result, the He abundance was the highest in the vicinity of the strongest magnetic pole which in that case was the pole of positive polarity. Oksala et al. (2015) studied the distribution of He, $\mathrm{C}, \mathrm{Si}$, and $\mathrm{Fe}$ on the surface of $\sigma$ Ori E. A large overabundant He spot was found to appear at the rotation phase 0.8 , but the location of the spot was not correlated with the position of the magnetic poles. The minimum abundances of $\mathrm{C}, \mathrm{Si}$, and $\mathrm{Fe}$ were found at the same phase where He showed the largest abundance. Finally, Kochukhov et al. (2011) studied the distribution of He on the surface of HD 37776 and concluded that the He concentration is at a maximum in the regions of maximum radial field. As only very few He-strong stars were studied with ZDI, future high-resolution, high signal-to-noise spectropolarimetric observations are needed to obtain a more complete picture on how the distribution of surface chemical spots is related to the magnetic field topology. We note that $\mathrm{CPD}-57^{\circ} 3509$ has a relatively low projected rotational velocity, and due to the presence of the $\mathrm{kG}$ magnetic field and the distinct inhomogeneous element abundance distribution, it can serve as an excellent laboratory to study various atmospheric effects that interact with the magnetic field. Further, the discovered pulsational variability on the time scale of tens of minutes has to be confirmed by future high-resolution spectroscopic time series.

\section{ACKNOWLEDGMENTS}

Based on observations made with ESO Telescopes at the La Silla Paranal Observatory under programmes 094.D0355 and 191.D-0255. The observations on Cerro Armazones are supported by the Nordrhein-Westfälische Akademie der Wissenschaften und der Künste in the framework of the academy program of the Federal Republic of Germany and the state Nordrhein-Westfalen. AK acknowledges financial support from RFBR grant 16-02-00604A.

\section{REFERENCES}

Altmann M., Röser S., Demleitner M., Bastian U., Schilbach E., 2017, A\&A, 600, L4

Angel J. R. P., Landstreet J. D., 1970, ApJ, 160, L147

Appenzeller I., et al., 1998, The ESO Messenger, 94, 1

Baume G., Vázquez R. A., Carraro G., Feinstein A., 2003, A\&A, 402, 549

Brown S. F., Donati J.-F., Rees D. E., Semel M., 1991, A\&A, 250, 463

Collier-Cameron A., Unruh Y. C., 1994, MNRAS, 269, 814

Dekker H., D'Odorico S., Kaufer A., Delabre B., Kotzlowski H., 2000, SPIE, 4008, 534

Dias W. S., Monteiro H., Caetano T. C., Lépine J. R. D., Assafin M., Oliveira A. F., 2014, A\&A, 564, A79

Gray R. O., Corbally C. J., 1994, AJ, 107, 742

ESA, 1997, The HIPPARCOS and Tycho catalogues, ESASP 1200

Evans C. J., et al., 2005, A\&A, 437, 467

Gaia Collaboration, et al., 2016a, A\&A, 595, A1

Gaia Collaboration, et al., 2016b, A\&A, 595, A2

Grunhut J. H., et al., 2012, MNRAS, 419, 1610

Hackman T., Jetsu L., Tuominen I., 2001, A\&A, 374, 171

Høg E., et al., 2000, A\&A, 355, L27

Hubrig S., Kurtz D. W., Bagnulo S., Szeifert T., Schöller M., Mathys G., Dziembowski W. A., 2004a, A\&A, 415, 661

Hubrig S., Szeifert T., Schöller M., Mathys G., Kurtz D. W., 2004b, A\&A, 415, 685

Hubrig S., North P., Schöller M., 2007, Astr. Nachr., 328, 475

Hubrig S., Schöller M., Kholtygin A. F., 2014a, MNRAS, 440, L6

Hubrig S., et al. 2014b, MNRAS, 440, L6

Hubrig S., Kholtygin A. F., Schöller M., Ilyin I., 2017, MNRAS, 467, L81 
Kharchenko N. V., Piskunov A. E., Röser S., Schilbach E., Scholz R.-D., 2004, Astr. Nachr., 325, 740

Kharchenko N. V., Piskunov A. E., Röser S., Schilbach E., Scholz R.-D., 2005, A\&A, 438, 1163

Kharchenko N. V., Piskunov A. E., Schilbach E., Röser S., Scholz R.-D., 2013, A\&A, 558, A53

Kochukhov O., Lundin A., Romanyuk I., Kudryavtsev D., 2011, ApJ, 726, 24

Korhonen H., et al., 2013, A\&A, 553, A27

Kupka F., Piskunov N. E., Ryabchikova T. A., Stempels H. C., Weiss W. W., 1999, A\&AS, 138, 119

Kurucz R. L., 1993, CD-ROM No. 13 (Cambridge, Mass: $\mathrm{SAO})$

Landstreet J. D., Mathys G., 2000, A\&A, 359, 213

Lindegren L., et al., 2016, A\&A, 595, A4

Makaganiuk V., et al., 2011, A\&A, 525, A97

Maeder A., Przybilla N., Nieva M.-F., Georgy C., Meynet

G., Ekström S., Eggenberger P., 2014, A\&A, 565, A39

Nieva M.-F., Przybilla N., 2012, A\&A, 539, A143

North P., Cramer N., 1984, A\&AS, 58, 387

North P., 1985, A\&A, 148, 165

Oksala M. E., et al., 2015, MNRAS, 451, 2015

Piskunov N. E., Tuominen I., Vilhu O., 1990, A\&A, 230, 363

Piskunov N. E., Kupka F., Ryabchikova T. A., Weiss

W. W., Jeffery C. S., 1995, A\&AS, 112, 525

Press W. H., Teukolsky S. A., Vetterling W. T., Flannery B. P., 1992, Numerical Recipes, 2nd edn. (Cambridge: Cambridge University Press)

Preston G. W., 1967, ApJ, 150, 547

Przybilla N., et al., 2016, A\&A, 587, A7

Ramolla M., et al., 2013, Astr. Nachr., 334, 1115

Rice J. B., Wehlau W. H., Holmgren D. E., 1997, A\&A, 326, 988

Rice J. B., Strassmeier K. G., 2000, A\&AS, 147, 151

Röser S., Demleitner M., Schilbach E., 2010, AJ, 139, 2440

Seber G. A. F., 1977, Linear Regression Analysis (New York: Wiley)

Sikora J., et al., 2015, MNRAS, 451, 1928

Skrutskie M. F., et al., 2006, AJ, 131, 1163

Stibbs D. W. N., 1950, MNRAS, 110, 395

Yakunin I., et al., 2015, MNRAS, 447, 1418

Zacharias N., Finch C. T., Girard T. M., Henden A., Bartlett J. L., Monet D. G., Zacharias M. I., 2013, AJ, 145,44

Zacharias N., Finch C., Frouard J., 2017, AJ, 153, 166

\section{APPENDIX A: THE MEMBERSHIP OF CPD $-57^{\circ} 3509$ IN NGC 3293}

The star $\mathrm{CPD}-57^{\circ} 3509$ is located only 66 arcsec from the cluster centre of NGC 3293, whereas the angular radii of the cluster core, of the central part of the cluster, and of the whole cluster were determined as 72 arcsec, 306 arcsec, and 486 arcsec, respectively Kharchenko et al. 2013). Although this relatively bright star is listed in the Tycho catalogue (ESA 1997), it has no proper motion measurement in the Tycho-2 catalogue (Høg et al. 2000). Therefore, it was not included in the catalogue of stars in open cluster areas of Kharchenko et al. (2004), where membership probabilities are given for 520 open clusters of the survey of Kharchenko et al. (2005) based on proper motions and optical photometry. In the new Milky Way Star Clusters (MWSC) survey by Kharchenko et al. (2013), based on proper motions from the PPMXL catalogue (Röser. Demleitner \& Schilbach 2010) and nearinfrared photometry from the Two Micron All Sky Survey (2MASS; Skrutskie et al. 2006), CPD $-57^{\circ} 3509$ is however listed with a $95 \%$ membership probability from proper motion and $99 \%$ from its $J K_{s}$ photometry. Dias et al. (2014) also determined a high (98\%) membership probability based only on proper motions from the fourth US Naval Observatory CCD Astrograph Catalog (UCAC4; Zacharias et al. 2013).

One should however note that the proper motion errors of the catalogues used for the above mentioned cluster membership studies are rather large. In case of CPD $-57^{\circ} 3509$ they are \pm 2.5 mas $/ y r$ in the PPMXL and between $\pm 2.2 \mathrm{mas} / \mathrm{yr}$ and $\pm 4.0 \mathrm{mas} / \mathrm{yr}$ in the UCAC4 catalogue. A significant improvement of PPMXL proper motions was recently achieved with the Hot Stuff for One Year (HSOY; Altmann et al. 2017) catalogue, which represents a new reduction of the PPMXL including Gaia DR1 data Gaia Collaboration et al. 2016a, b; Lindegren et al. 2016). The new UCAC5 catalogue (Zacharias, Finch \& Frouard 2017) presents very accurate proper motions combining the UCAC positions with those from Gaia DR1. Because CPD $-57^{\circ} 3509$ was not in the Tycho- 2 catalogue, it was not included in the Tycho-Gaia Astrometric Solution (TGAS) of Gaia DR1. However, its HSOY proper motion, $\mu_{\alpha} \cos \delta=$ $-5.9 \pm 1.1 \mathrm{mas} / \mathrm{yr}, \mu_{\delta}=+3.5 \pm 1.1 \mathrm{mas} / \mathrm{yr}$, and its UCAC5 proper motion, $-6.1 \pm 0.9 \mathrm{mas} / \mathrm{yr},+3.2 \pm 0.9 \mathrm{mas} / \mathrm{yr}$, are in very good agreement with the mean TGAS proper motion of four of the five $1 \sigma$ members from Kharchenko et al. (2004) that can be found in TGAS: $-6.7 \pm 1.2 \mathrm{mas} / \mathrm{yr}$, $+3.7 \pm 0.3 \mathrm{mas} / \mathrm{yr}$. Their standard deviation in $\mu_{\alpha} \cos \delta$ is still about three times larger than in $\mu_{\delta}$ but was even much larger before we excluded one of the five stars as an outlier.

We do not consider the TGAS parallaxes of the few $1 \sigma$ members from Kharchenko et al. (2004), as they show a large spread, and since $\mathrm{CPD}-57^{\circ} 3509$ is not included in the TGAS. The very large standard deviation of the parallaxes of the five $1 \sigma$ members $( \pm 0.77$ mas) reduces to \pm 0.19 mas, if we again exclude the same one outlier. However, the generally assumed additional systematic error of \pm 0.3 mas in TGAS parallaxes (Gaia Collaboration et al. 2016b) leads to a $60 \%$ relative distance uncertainty of a cluster at about $2 \mathrm{kpc}$ distance. According to Kharchenko et al. (2005, 2013), the distance to NGC 3293 is about 2400 pc.

Concerning the radial velocity of $\mathrm{CPD}-57^{\circ} 3509$, there is one measurement, $-16 \mathrm{kms}^{-1}$ given without an error estimate (Evans et al. 2005), and slight variability $-16 \ldots-20 \mathrm{~km} \mathrm{~s}^{-1}$ measured by Przybilla et al. (2016) due to the presence of spots. This is in reasonably good agreement with the mean cluster radial velocities determined by Kharchenko et al. (2005, 2013) and Evans et al. (2005), of $-12.3 \pm 2.3 \mathrm{~km} \mathrm{~s}^{-1},-11.2 \pm 2.1 \mathrm{~km} \mathrm{~s}^{-1}$, and $-12 \pm 5 \mathrm{~km} \mathrm{~s}^{-1}$, respectively.

Thus the membership of $\mathrm{CPD}-57^{\circ} 3509$ in the cluster NGC 3293 is based on the star's projection to the cluster core, and its available proper motion, photometry, and radial velocity. Gaia DR2, expected for April 2018, will not 
Table B1. Differential photometry for $\mathrm{CPD}-57^{\circ} 3509$ in the $B$ band.

\begin{tabular}{ccrcc}
\hline \hline MJD & Airmass & $\begin{array}{c}\Delta m_{\mathrm{B}} \\
{[\mathrm{mag}]}\end{array}$ & $\phi_{\text {hyd }}$ & $\phi_{\text {all }}$ \\
\hline 56754.66893 & 1.216 & 0.0065 & 0.025 & 0.028 \\
56755.71689 & 1.205 & -0.0058 & 0.189 & 0.193 \\
56756.62353 & 1.277 & -0.0057 & 0.332 & 0.336 \\
56763.67501 & 1.201 & -0.0069 & 0.440 & 0.444 \\
56764.59286 & 1.298 & 0.0029 & 0.585 & 0.588 \\
56765.67003 & 1.201 & 0.0020 & 0.754 & 0.758 \\
56766.59765 & 1.274 & 0.0068 & 0.900 & 0.903 \\
56767.65829 & 1.201 & -0.0042 & 0.067 & 0.070 \\
56768.59115 & 1.276 & 0.0010 & 0.213 & 0.217 \\
56772.58155 & 1.274 & 0.0071 & 0.841 & 0.844 \\
56773.68069 & 1.213 & 0.0049 & 0.013 & 0.017 \\
56775.66947 & 1.209 & -0.0061 & 0.326 & 0.329 \\
56778.58729 & 1.235 & 0.0053 & 0.785 & 0.788 \\
56783.64584 & 1.208 & -0.0103 & 0.580 & 0.583 \\
56785.60891 & 1.202 & -0.0010 & 0.889 & 0.891 \\
56787.64327 & 1.214 & -0.0027 & 0.208 & 0.211 \\
56796.55959 & 1.211 & -0.0041 & 0.610 & 0.612 \\
56797.61941 & 1.217 & 0.0043 & 0.777 & 0.779 \\
56805.56942 & 1.201 & 0.0083 & 0.027 & 0.028 \\
56806.50446 & 1.244 & -0.0072 & 0.174 & 0.175 \\
57112.53783 & 1.831 & -0.0188 & 0.285 & 0.275 \\
57112.59368 & 1.460 & -0.0143 & 0.294 & 0.283 \\
57113.53480 & 1.834 & -0.0020 & 0.442 & 0.431 \\
57113.55310 & 1.682 & -0.0005 & 0.445 & 0.434 \\
57113.58957 & 1.466 & -0.0163 & 0.450 & 0.440 \\
57113.60865 & 1.386 & -0.0136 & 0.453 & 0.443 \\
57113.62245 & 1.339 & -0.0073 & 0.456 & 0.445 \\
57113.64136 & 1.288 & -0.0179 & 0.458 & 0.448 \\
57113.65530 & 1.258 & -0.0119 & 0.461 & 0.450 \\
57114.53666 & 1.792 & -0.0029 & 0.599 & 0.589 \\
57114.59523 & 1.428 & 0.0005 & 0.608 & 0.598 \\
57114.68590 & 1.214 & -0.0111 & 0.623 & 0.612 \\
57115.54074 & 1.735 & -0.0004 & 0.757 & 0.747 \\
57115.59857 & 1.404 & -0.0007 & 0.766 & 0.756 \\
57115.68972 & 1.209 & 0.0048 & 0.781 & 0.770 \\
\hline & & & &
\end{tabular}

only provide even more accurate proper motion membership probabilities but also enable a membership study using individual stellar parallaxes of many more stars in the cluster area.

\section{APPENDIX B: PHOTOMETRIC DATA}

Tables B1 and B2 present the photometric data of CPD $-57^{\circ} 3509$, where the Modified Julian Date, the airmass of the observation, the differential photometric values $\Delta m_{\mathrm{B}}$ and $\Delta m_{\mathrm{V}}$, and the phase information $\phi_{\mathrm{hyd}}$ and $\phi_{\text {all }}$ are given.
Table B2. Differential photometry for CPD $-57^{\circ} 3509$ in the $V$ band.

\begin{tabular}{crrrr}
\hline \hline MJD & Airmass & $\begin{array}{c}\Delta m_{\mathrm{V}} \\
{[\mathrm{mag}]}\end{array}$ & $\phi_{\text {hyd }}$ & $\phi_{\text {all }}$ \\
& & & \\
\hline 56754.67501 & 1.211 & 0.0004 & 0.025 & 0.029 \\
56755.72287 & 1.208 & -0.0058 & 0.190 & 0.194 \\
56756.62949 & 1.265 & -0.0071 & 0.333 & 0.337 \\
56763.68112 & 1.201 & -0.0062 & 0.441 & 0.445 \\
56764.59909 & 1.283 & 0.0040 & 0.586 & 0.589 \\
56765.87662 & 1.201 & -0.0050 & 0.786 & 0.790 \\
56766.60432 & 1.261 & 0.0064 & 0.901 & 0.904 \\
56767.66445 & 1.201 & 0.0021 & 0.068 & 0.071 \\
56768.59730 & 1.264 & -0.0066 & 0.214 & 0.218 \\
56772.58781 & 1.261 & 0.0017 & 0.842 & 0.845 \\
56775.67570 & 1.213 & 0.0011 & 0.327 & 0.330 \\
56778.59372 & 1.226 & 0.0022 & 0.786 & 0.789 \\
56783.65240 & 1.212 & -0.0061 & 0.581 & 0.584 \\
56785.61510 & 1.201 & 0.0036 & 0.890 & 0.892 \\
56796.56586 & 1.207 & -0.0004 & 0.611 & 0.613 \\
56806.51094 & 1.235 & -0.0014 & 0.175 & 0.176 \\
57112.54384 & 1.777 & -0.0144 & 0.286 & 0.276 \\
57112.59944 & 1.434 & -0.0207 & 0.295 & 0.284 \\
57113.54069 & 1.781 & -0.0143 & 0.443 & 0.432 \\
57113.58178 & 1.504 & -0.0218 & 0.449 & 0.439 \\
57113.59579 & 1.438 & -0.0146 & 0.451 & 0.441 \\
57113.61466 & 1.365 & -0.0299 & 0.454 & 0.444 \\
57113.62857 & 1.321 & -0.0217 & 0.456 & 0.446 \\
57113.64753 & 1.274 & -0.0263 & 0.459 & 0.449 \\
57113.66151 & 1.247 & -0.0220 & 0.462 & 0.451 \\
57114.54293 & 1.740 & -0.0176 & 0.600 & 0.590 \\
57114.60158 & 1.403 & 0.0112 & 0.609 & 0.599 \\
57114.69188 & 1.209 & -0.0111 & 0.624 & 0.613 \\
57115.60458 & 1.381 & -0.0086 & 0.767 & 0.757 \\
57115.69598 & 1.205 & 0.0121 & 0.782 & 0.771 \\
\hline & & & &
\end{tabular}

